\title{
Ultrasound assessment of tensile stress in carotid arteries of healthy human subjects with varying age
}

\author{
Xianghong Luo', Lianfang $\mathrm{Du}^{2}$ and Zhaojun $\mathrm{Li}^{2^{*}}$
}

\begin{abstract}
Background: Arterial remodeling is thought to reflect the adaptation of the vessel wall to mechanical and hemodynamic stimuli and contributes to the progression of cardiovascular and cerebrovascular diseases. Tensile stress (TS) is one of the mechanical properties of the artery wall. The purpose of this study was to investigate the tensile stress change (TS) of carotid artery with varying viscoelasticity in healthy subjects within two groups of different ages.
\end{abstract}

Methods: Forty-five subjects were recruited and randomly assigned into the group at the age above 50 years and below 50 years. The carotid arteries were examined by ultrasonography, using the techniques of shear wave elastography (SWE), shear wave dispersion (SWD) and radiofrequency (RF) -based ultrasound. The following values, including elastic modulus (SWER) and viscous index (SWDR), as well as the peak and mean TS of the left and right carotid arteries (L-PTS, R-PTS, L-MTS and R-MTS) were measured. The correlations between SWER, SWDR and tensile stress were evaluated.

Results: The $S W E_{R}$ and $S W D_{R}$ of carotid arteries are lower in the subjects $\geq 50$ years old than the subjects younger than 50 years $\left(S_{W E}, 10.29 \pm 9.57 \mathrm{kPa}\right.$ VS $17.24 \pm 14.07 \mathrm{kPa} ; \mathrm{SWD}_{\mathrm{R}}, 11.99 \pm 3.51(\mathrm{~m} / \mathrm{s}) / \mathrm{kHz}$ VS $13.97 \pm 3.71(\mathrm{~m} / \mathrm{s}) / \mathrm{kHz}$, $P<0.05)$. The R-PTS was lower in the group with younger age $(P<0.05)$. Pearson correlation analysis showed that $S W E_{R}$ of carotid artery was positively correlated with the parameters of tensile stress, R-PTS, R-MTS, L-PTS and LMTS $(r=0.218, r=0.359, r=0.209$ and $r=0.369$, respectively, $P<0.05)$. However, SWD $D_{R}$ of carotid arteries was not significantly associated with TS.

Conclusion: Ultrasonic shear wave imaging could be used to quantitatively assess carotid viscoelasticity. The carotid TS was related to its elasticity while little related to its viscosity, suggesting that mechanical properties of the arterial wall might be better revealed.

Trial registration: Date of our trial registration: 2018-06-11. Registered with the official website of China Clinical Trial Registration Center (ChiCTR1800016590)

Keywords: Shear wave dispersion, Shear wave elastography, Tensile stress, Carotid artery, Viscoelasticity, Ultrasonography

\footnotetext{
* Correspondence: Izj_197506@126.com

${ }^{2}$ Department of Ultrasound, Shanghai General Hospital, Shanghai Jiao Tong University School of Medicine, 100 Haining Road, Hongkou District, Shanghai 200080, China

Full list of author information is available at the end of the article
}

(c) The Author(s). 2019 Open Access This article is distributed under the terms of the Creative Commons Attribution 4.0 International License (http://creativecommons.org/licenses/by/4.0/), which permits unrestricted use, distribution, and reproduction in any medium, provided you give appropriate credit to the original author(s) and the source, provide a link to the Creative Commons license, and indicate if changes were made. The Creative Commons Public Domain Dedication waiver (http://creativecommons.org/publicdomain/zero/1.0/) applies to the data made available in this article, unless otherwise stated. 


\section{Background}

Cardiovascular disease is still a major cause of morbidity and mortality in the world. It is essential to recognize early changes in vascular function and morphology to help identify individuals at risk for cardiovascular disease. Arterial remodeling or dilatation of carotid arteries has been shown to be related to increased mechanical stress from high pulsatile loads, leading to breakdown of elastic fibers within the arterial wall $[1,2]$. Arterial remodeling is a potentially important pathophysiologic change in the development of atherosclerosis, which acts to counteract the development of lumen compromise of large artery by mutual adaptation of diameter to wall thickening [3]. Carotid geometry and wall tensile stress can be evaluated at the carotid artery, because its simple geometry allows the application of Laplace's law for wall stress estimation. Hypertension increases the tensile stress applied on the carotid artery, thus carotid intimamedia thickness (IMT) and stiffness, and favors atherosclerotic plaque progression [4].

The arterial wall has been known as one of the most intricate structures and presents a certain biomechanical property of viscoelasticity [5]. This behavior presents a non-linear mechanical relationship and attributes in part to fluid transport within the solid matrix [6, 7]. However, most of the arterial TS had been measured through biomechanical experiments in vitro, and little is known about the nature of the adjustments to the arterial viscoelasticity, which corresponds to the altered wall TS.

In recent years, shear wave-based elastography techniques, such as shear wave elastography (SWE) and shear wave dispersion (SWD) have received wide attention for noninvasive assessment of elasticity and viscidity properties $[8,9]$. This study was to assess the carotid TS and viscoelasticity by ultrasonic technologies and explored the relationship between them.

\section{Methods}

\section{Study design and setting participants}

The study was registered as a clinical trial (ChiCTR1800016590, 2018-06-11) approved by our Institutional Ethics Review Board (2017KY009). Written informed consent was obtained from all participants in this study.

Forty-five healthy subjects were enrolled from August 1, 2017 to May 1, 2018. They were selected based on the electronic medical records. Participants that have active bleeding, history of cardiovascular or cerebrovascular events, vascular diseases of the extremities, immune diseases, severe liver, lung, kidney diseases, or malignant tumor were excluded. They were divided into 2 groups according to their ages: group A ( $\geq 50$ years) and group B ( $<50$ years). Blood pressure was measured in the supine position after $10 \mathrm{~min}$ of rest and defined as the average of 3 consecutive systolic blood pressure (SBP) and diastolic blood pressure (DBP). Blood samples were obtained for the measurements of glucose, high density lipoprotein (HDL), triglycerides and low-density lipoprotein-cholesterol (LDL) after $12 \mathrm{~h}$ of fasting.

\section{Carotid artery B-mode ultrasound}

After high-resolution common carotid artery (CCA) images were obtained, the viscoelasticity of the CCA was measured using an Aplio 900 ultrasound system (Canon Medical Systems Corporation, Otawara, Japan) equipped with PVI-475BX curved abdominal transducer (frequency range: $1-8-\mathrm{MHz}$ and mid frequency: $5.0 \mathrm{MHz}$ ) [10]. The ultrasound image was frozen at electrocardiographic end diastole (R-wave) by electrocardiogram (ECG) triggering. Five $2 \mathrm{~mm}$ circular regions of interest (ROI) were selectively placed on anterior and posterior walls of bilateral carotid arteries $(1 \mathrm{~cm}$ proximal to the carotid bifurcation). The motion of vascular wall for 10 20 cardiac cycles was recorded and measurements were taken during the systolic phase. Shear wave elastic modulus $\left(\mathrm{SWE}_{\mathrm{R}}\right)$ and shear wave dispersion $\left(\mathrm{SWD}_{\mathrm{R}}\right)$ were measured after the $\mathrm{R}$ wave of the ECG. The arterial shear wave profiles, including the elastic map (Fig. 1a), propagation map (Fig. 1b), two-dimensional reference map (Fig. 1c) and shear wave dispersion map (Fig. 1d) were obtained with single-shot acquisition and displayed on QuadView.

The carotid structure and tensile stress were measured using Mylab Twice ultrasound system (Esaote, Firenze, Italy) equipped with a LA523 linear transducer (4-13 MHz) and the software, which enables the analysis of radiofrequency-based ultrasound images. The common carotid intima-media thickness (CIMT) and inner diameter (CCID) were measured by radiofrequency-based ultrasound tracking of the carotid wall displacement for at least six cardiac cycles and the mean values were calculated automatically by the software. The carotid peak tensile stress (PTS) and mean tensile stress (MTS) were calculated according to Laplace's law using the equations: (PTS $=\mathrm{SBP} \times\left(\mathrm{CCID}_{\mathrm{R}} / 2\right)$ / CIMT $(\mathrm{mmHg}), \mathrm{MTS}=\mathrm{MBP} \times\left(\mathrm{CCID}_{\mathrm{T}} / 2\right) / \mathrm{CIMT}(\mathrm{mmHg})$ [11]. SBP was systolic blood pressure, while MBP was the mean blood pressure and calculated by $(\mathrm{SBP}+2 \times \mathrm{DBP}) / 3$. $\mathrm{CCID}_{\mathrm{R}}$ was the inner diameter of common carotid artery at the end of systolic phase, while $\mathrm{CCID}_{\mathrm{T}}$ was the inner diameter of common carotid artery at the end of diastolic phase. 1dyne $/ \mathrm{cm}^{2}=7.5 \times 10^{-4} \mathrm{mmHg}$.

\section{Statistical analyses}

All statistical analyses were performed using SPSS 13.0 statistical analysis software. The continuous variables were expressed as mean \pm SD and compared using Student's $t$-tests, while categorical variables were expressed as percentages and compared using chi-squared test. 


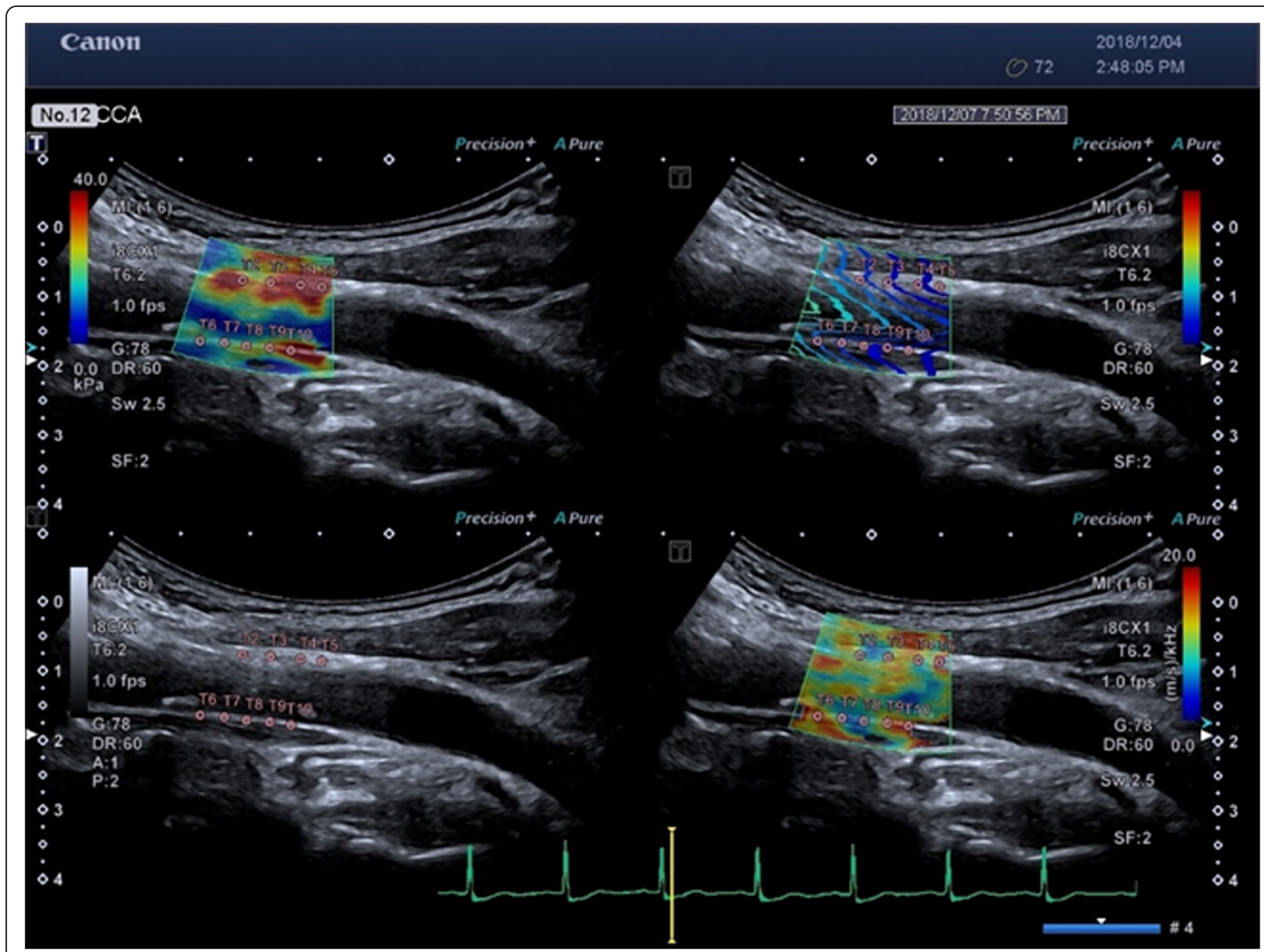

Fig. 1 The arterial shear wave profiles were displayed by. QuadView, including 4 maps for: a elastic map, b propagation map, c two-dimensional reference map and $\mathbf{d}$ shear wave dispersion map.

Agreement of measurement was evaluated by BlandAltman plots. Pearson correlation analysis was used to evaluate the correlation between carotid viscoelasticity and TS. $P<0.05$ was considered statistically significant.

\section{Results}

\section{Clinical characteristics}

The baseline characteristics of two age groups were summarized in Table 1 . The mean height of the subjects in group $\mathrm{A}$ is less than that of group A. The mean SBP and DBP were higher in group A (all $P<0.05$ ) than group B. No significant difference of gender ratio, weight, body mass index, fasting glucose, TG, TC, LDL$\mathrm{C}$ was found between the two groups (all $P>0.05$ ).

\section{Geometry, viscoelasticic feature and tensile stress of carotid arteries in two groups}

The $S W E_{R}$ and $S W D_{R}$, which are the two parameters that represent the carotid viscoelasticity, were significantly lower in the group A than those in the group B $(P=0.040$ and 0.043 , respectively). The bilateral CIMT, $\mathrm{CCID}_{\mathrm{T}}$ and $\mathrm{CCID}_{\mathrm{R}}$, which are used to characterize the geometry of the carotid arteries, were all higher in the group of subjects older than 50 years (all $P<0.05$ ). The mean PTS of the right carotid arteries was lower in the group A $(P<$ 0.05 ), while no significant differences of the PTS of left carotid arteries and bilateral MTS were found between the two groups (all $P>0.05$ ) (Table 2).

\section{Correlation between the carotid viscoelasticity and tensile} stress

Figure 2 and Table 3 showed that $\mathrm{SWE}_{\mathrm{R}}$ has a positive correlation with R-PTS, R- MTS, L-PTS and L-MTS $(r=0.218,0.359,0.209,0.369$, respectively, all $P<0.05)$, whereas no correlation was found between the $S W D_{R}$ and the parameters of TS (all $P>0.05$ ).

\section{Reproducibility analysis}

A week later, a total of 22 subjects were randomly selected and involved in the reproducibility evaluation. 
Table 1 Characteristics of two age groups

\begin{tabular}{|c|c|c|c|c|}
\hline Variable & $\begin{array}{l}\text { Group A } \\
(n=23)\end{array}$ & $\begin{array}{l}\text { Group B } \\
(n=22)\end{array}$ & $t / x^{2}$ & $P$ \\
\hline Gender (F/M) & $11 / 12$ & $11 / 11$ & 0.023 & 0.879 \\
\hline Height, cm & $162.9 \pm 7.1$ & $167.5 \pm 7.9$ & -2.301 & 0.025 \\
\hline Weight, kg & $68.3 \pm 17.3$ & $66.7 \pm 15.6$ & 0.348 & 0.729 \\
\hline Body mass index, $\mathrm{kg} / \mathrm{m}^{2}$ & $25.8 \pm 7.4$ & $23.5 \pm 4.1$ & 1.444 & 0.154 \\
\hline SBP, mmHg & $139.6 \pm 11.1$ & $125.7 \pm 9.1$ & 5.102 & $<0.001$ \\
\hline $\mathrm{DBP}, \mathrm{mmHg}$ & $87.9 \pm 7.2$ & $79.7 \pm 6.1$ & 4.607 & $<0.001$ \\
\hline Glucose, $\mathrm{m} \mathrm{mol} / \mathrm{L}$ & $6.1 \pm 1.61$ & $5.2 \pm 1.21$ & 1.001 & 0.327 \\
\hline Total cholesterol, mmol /L & $4.6 \pm 1.11$ & $4.4 \pm 0.81$ & 0.763 & 0.456 \\
\hline Triglycerides, mmol/L & $1.7 \pm 1.31$ & $1.2 \pm 0.81$ & 1.12 & 0.275 \\
\hline Low-density lipoprotein, mmol/L & $2.9 \pm 1.01$ & $2.7 \pm 0.81$ & 0.77 & 0.433 \\
\hline Diabetes mellitus ( $n$ ) & 3 & 1 & 0.156 & 0.693 \\
\hline Hypertension (n) & 5 & 3 & 0.044 & 0.833 \\
\hline
\end{tabular}

$1 \mathrm{mmHg}=0.133 \mathrm{kPa}$

Table 2 Comparison of carotid viscoelasticity, structure and tensile stress between groups

\begin{tabular}{|c|c|c|c|c|}
\hline Variable & $\begin{array}{l}\text { Group A } \\
(n=23)\end{array}$ & $\begin{array}{l}\text { Group B } \\
(n=22)\end{array}$ & $t$ & $P$ \\
\hline \multicolumn{5}{|l|}{ Viscoelasticity } \\
\hline $\mathrm{SWE}_{\mathrm{R}} \mathrm{kPa}$ & $10.29 \pm 9.57$ & $17.24 \pm 14.07$ & -2.236 & 0.040 \\
\hline $\mathrm{SWD}_{\mathrm{R}}(\mathrm{m} / \mathrm{s}) / \mathrm{kHz}$ & $11.99 \pm 3.51$ & $13.97 \pm 3.71$ & -2.129 & 0.043 \\
\hline \multicolumn{5}{|l|}{ Structure } \\
\hline R-CIMT $(\mu \mathrm{m})$ & $677.6 \pm 138.4$ & $449.1 \pm 131.9$ & 6.321 & $<0.001$ \\
\hline L-CIMT ( $\mu \mathrm{m})$ & $674.7 \pm 119.8$ & $503.9 \pm 193.2$ & 3.976 & $<0.001$ \\
\hline $\mathrm{R}-\mathrm{CCID}$ ( $(\mathrm{mm})$ & $8.23 \pm 0.89$ & $6.43 \pm 0.40$ & 5.946 & $<0.001$ \\
\hline $\mathrm{L}-\mathrm{CCID}$ ( $(\mathrm{mm})$ & $7.93 \pm 0.99$ & $6.50 \pm 0.41$ & 7.575 & $<0.001$ \\
\hline $\mathrm{R}-\mathrm{CCID}_{\mathrm{R}}(\mathrm{mm})$ & $8.57 \pm 1.03$ & $7.00 \pm 0.55$ & 7.095 & $<0.001$ \\
\hline $\mathrm{L}-\mathrm{CCID}_{\mathrm{R}}(\mathrm{mm})$ & $8.20 \pm 1.09$ & $7.04 \pm 0.56$ & 5.000 & $<0.001$ \\
\hline $\mathrm{R}-\mathrm{CCAD}(\mu \mathrm{m})$ & $339.9 \pm 142.7$ & $571.1 \pm 148.2$ & -5.946 & $<0.001$ \\
\hline L-CCAD $(\mu \mathrm{m})$ & $273.6 \pm 98.6$ & $535.4 \pm 153.9$ & -7.575 & $<0.001$ \\
\hline \multicolumn{5}{|l|}{ Tensile stress } \\
\hline R-PTS (mmHg) & $920.4 \pm 160.3$ & $1073.6 \pm 285.8$ & -2.474 & 0.017 \\
\hline R-MTS (mmHg) & $783.8 \pm 189.9$ & $884.4 \pm 224.0$ & -1.812 & 0.076 \\
\hline L-PTS (mmHg) & $648.1 \pm 117.4$ & $723.8 \pm 195.5$ & -1.758 & 0.084 \\
\hline L-MTS (mmHg) & $600.9 \pm 108.2$ & $644.6 \pm 168.2$ & -1.156 & 0.253 \\
\hline
\end{tabular}

$S W E_{R}$ Shear wave elastic modulus in electrocardiographic $\mathrm{R}$ wave, $S W D_{R}$ Shear wave dispersion in electrocardiographic $\mathrm{R}$ wave, $L-C I M T$ Left common carotid intima-media thickness, $R$-CIMT Right common carotid intima-media thickness; $L-C C I D_{T}$ and $R-C C I D_{T}$ Left and right common carotid inners at end of diastole, respectively, $L-C C I D_{R}$ and $R-C C I D_{R}$ Left and right common carotid inner diameters at end of systole, respectively, L-CCAD and R-CCAD Difference of CCID between diastole and systole on left and right, R-PTS and R-MTS Right carotid peak tensile stress and mean tensile stress, L-PTS and L-MTS Left carotid peak tensile stress and mean tensile stress

1 dyne $/ \mathrm{cm}^{2}=7.5 \times 10^{-4} \mathrm{mmHg}$
$\mathrm{SWD}_{\mathrm{R}}$ and $\mathrm{SWE}_{\mathrm{R}}$ were measured repeatedly by the same investigator. The Bland-Altman plots showed the good agreement between the parameters obtained by two separate measurements (Fig. 3).

\section{Discussion}

The cardiovascular and cerebrovascular diseases are still the number 1 cause of death globally. However, it is known that the onset and progression of these diseases can be predicted by the biomechanical features of large arteries, such as carotid arteries. Therefore, some of the predictors, such as mechanical stress and strain are very important for identifying the potential risk over the processes of arterial pathologies. TS, a parameter derived from certain measurements by using Laplace's law, is associated with the inflation pressure, wall thickness and inner diameter [12]. In addition, TS of arterial wall is significantly related to the viscoelasticity of arteries. Our study shows that $S W E_{R}$ and $S W D_{R}$, the two parameters that represent the viscoelasticity of arteries were lower in the subjects older than 50 years that those of the younger subjects. We also found that no significant difference of PTS and MTS exist between the two groups, but the PTS of the right carotid arteries in the group with older ages was lower than the younger ones. The PTS and MTS, were positively corelated with $S W E_{R}$, but no correlation was found between PTS and $\mathrm{SWD}_{\mathrm{R}}$, which implys that the arterial elasticity may have some implication in the change of tensile stress.

The geometry changes of the carotid artery are closely related to the mechanical properties. CIMT, which is usually measured by noninvasive ultrasound, is strongly associated with cardiovascular and cerebrovascular events and used for evaluating progression: regression of atherosclerosis and for predicting arterial remolding and 

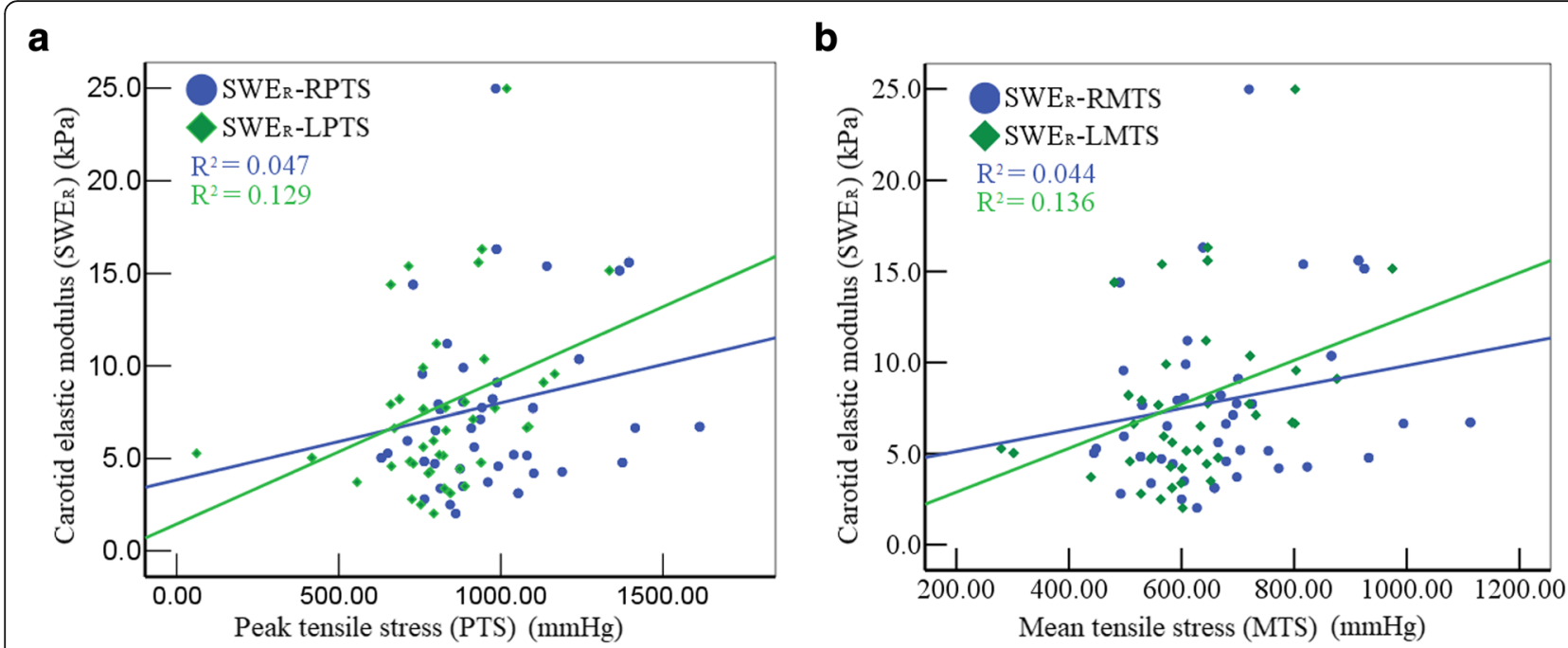

Fig. 2 Correlation between the carotid viscoelasticity and tensile stress. a SWE $E_{R}$ correlated positively with right and left carotid peak tensile stress, respectively. $\mathbf{b} S W E_{R}$ correlated positively with right and left carotid mean tensile stress, respectively

subsequent clinical complications [13, 14]. We measured the CIMT using radiofrequency-based ultrasound technique. The two-dimensional vascular structure can be clearly visualized, which allows the real-time measurement of CIMT within six cardiac cycles, with a resolution of $10 \mu \mathrm{m}$. This technology a reliable method for the clinical evaluation of arterial structure and function [15]. Our study shows that the CIMT increased in the subjects older than 50 years, suggesting that the carotid arterial remolding has some relationship with ages. CIMT measurements respectively made on the left and right carotid arteries could represent separate phenotypes because their patterns of associations with risk factors are different. For example, on the left carotid artery, the CIMT is thicker and shows stronger associations with blood lipid, glucose and lower estrogens; while on the right carotid artery, the CIMT is thinner and significantly related to hemodynamics, such as hypertension and heart rate. These results suggest that the weights of risk factors are different on left and right carotid arteries $[16,17]$. In this study, the CCID was enlarged in the older ones, while CCAD, i.e. the difference of CCID between diastole and systole was decreased. These results also exhibited that carotid artery occurred remodeling with age.

The study by Carallo et al. [18] demonstrated that the circumferential wall tension (WT) of carotid arteries significantly increased with age. Conversely, the present results showed the TS did not parallel the increase in CIMT and CCID. The PTS of right carotid arteries is lower in the group with older ages, and no remarkable difference was found for the parameters of bilateral MTS and left PTS. Various reasons for participating in that: (1) Mechanical models of artery, such as WT and TS, derived from Laplace's law can be used to relate the arterial inner radius $(r)$ and internal pressure $(P)$. The WT was defined as $P \times r$ [19]. The WT model assumes a very thin wall, and then handles the pressure, which does not take into account wall thickness. The TS, being a corrected Laplace model, was $P \times r / C I M T$. TS could reflect the tensile response in circumference [11]. (2) The mechanical stretch can induce structural changes in the arterial wall, including VSMC hyperplasia and hypertrophy, as well as increased deposition of ECM collagen and elastin and result in arterial remodeling [20, 21]. On the other hand, the arterial remodeling could act on its

Table 3 Correlation analysis between carotid viscoelasticity tensile stress $[r(P)]$

\begin{tabular}{|c|c|c|c|c|c|c|}
\hline \multirow[t]{2}{*}{ Variable } & \multicolumn{3}{|l|}{$S W E_{R}$} & \multicolumn{3}{|l|}{$S W D_{R}$} \\
\hline & All subjects & Group A & Group B & All subjects & Group A & Group B \\
\hline R-PTS & $0.218(0.021)$ & $0.137(0.313)$ & $0.159(0.241)$ & $0.096(0.314)$ & $-0.404(0.002)$ & $0.172(0.204)$ \\
\hline R-MTS & $0.359(0.001)$ & $0.276(0.040)$ & $0.353(0.008)$ & $0.054(0.568)$ & $0.068(0.619)$ & $-0.062(0.650)$ \\
\hline L-PTS & $0.209(0.027)$ & $0.196(0148)$ & $0.142(0.298)$ & $0.067(0.485)$ & $-0.352(0.008)$ & $0.153(0.259)$ \\
\hline L-MTS & $0.369(0.001)$ & $0.415(0.001)$ & $0.313(0.019)$ & $0.005(0.956)$ & $0.086(0.530)$ & $-0.098(0.474)$ \\
\hline
\end{tabular}

$S W E_{R}$ Shear wave elastic modulus in electrocardiographic $\mathrm{R}$ wave, $S W D_{R}$ Shear wave dispersion in electrocardiographic $\mathrm{R}$ wave, $R$-PTS and R-MTS Right carotid peak tensile stress and mean tensile stress, L-PTS and L-MTS Left carotid peak tensile stress and mean tensile stress 


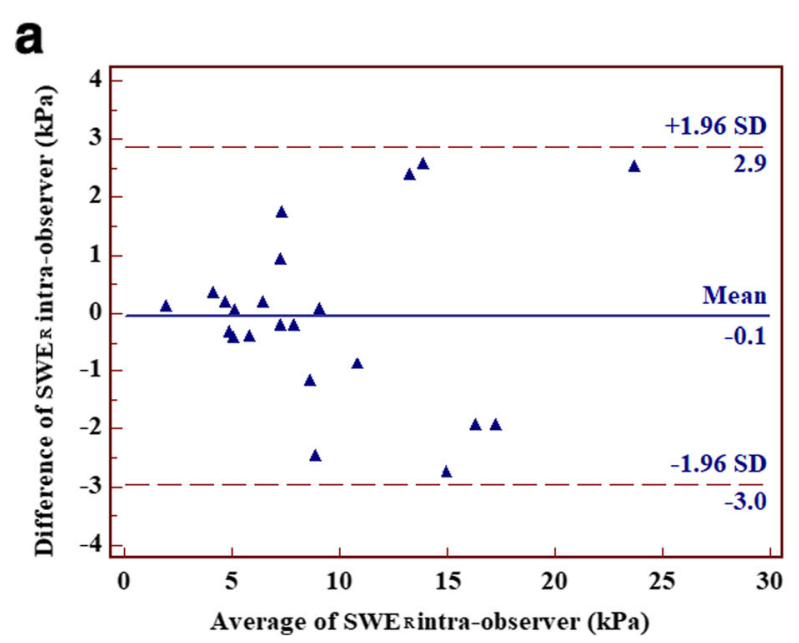

b

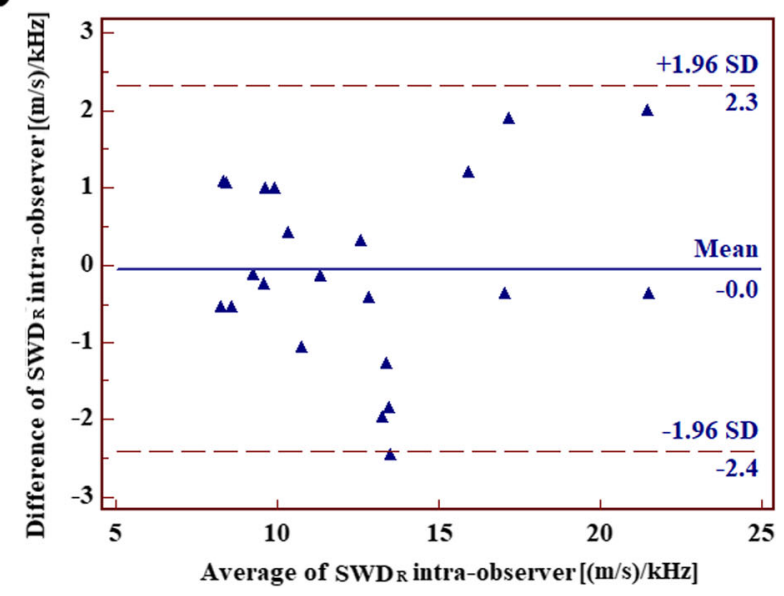

Fig. 3 Bland-Altman plots for agreement in $\operatorname{SWE}_{R}(\mathbf{a})$ and $\mathrm{SWD}_{R}(\mathbf{b})$ measurement

mechanical properties $[22,23]$. (3) The arterial tissue is viscoelasticity and show non-linear.

However, the arteries are of viscoelastic properties and exhibit the nonlinear stress-stain relations [5]. Several new non-invasive techniques have been used to study arterial elasticity, such as dimensional speckle-tracking imaging [24], ultrasonic radiofrequency tracking [25] and shear wave elastography [26-28]. However, in vivo, it is difficult to study the arterial viscidity due to its complex temporal changing behavior. Shear wave elasticity imaging may noninvasively evaluate the properties of soft tissues based on a group shear wave speed assuming that tissue is elastic; however, soft tissues are known to be viscoelastic, meaning the shear wave speed is dependent on the wave's frequency content. Over the last years, there has been significant innovation in the area of describing the viscoelastic properties of soft tissue by the frequency-dependent: shear wave dispersion (SWD, the change in speed with frequency) [29]. In this work, the viscoelastic properties of carotid artery were evaluated by SWD. The $\mathrm{SWE}_{R}$ and $\mathrm{SWD}_{\mathrm{R}}$ decreased with age. In addition, the TSs were positively connected with $\mathrm{SWE}_{\mathrm{R}}$, while were not related to $\mathrm{SWD}_{\mathrm{R}}$. This suggested that the arterial elasticity contributed its mechanical behavior rather than viscidity. The vascular smooth muscle cells, extracellular matrix proteins collagen and elastin play a crucial role in the viscoelastic properties, i.e. their spatial organization and interaction dominate the macroscopic non-linear vessel properties $[5,30]$. Higher vascular stiffness is typically found in older subjects because the elastic lamellae decreases with age, while the connective tissue and collagen fibers increase [31]. The mechanical characteristics of arteries were related to local pathologies of the arterial system, while wall viscosity change reflects a more general influence of age and diseases [32].

There are limitations for this study. We included a small sample size of 45 subjects in this study. In addition, the curved abdominal transducer was used to evaluate the carotid viscoelasticity, while transducer of linear array could provide better images and measurements. We only include the subjects with healthy carotid arteries. In the future, we will explore the value of using this technique to characterize the tensile stress features of the carotid arteries with pathology, such as atherosclerosis.

\section{Conclusion}

Ultrasonic shear wave imaging could be used to quantitatively assess carotid viscoelasticity. The tensile stress of carotid arteries is closely related to the elastic properties of aging carotid arteries, but no apparent correlation with the viscosity of carotid arteries, suggesting that mechanical properties of the arterial wall with age might be better revealed.

\section{Abbreviations}

CCA: Common carotid artery; CCID: Common carotid inner diameter; CIMT: Common carotid intima-media thickness; DBP: Diastolic blood pressure; LDL: Low-density lipoprotein-cholesterol; MTS: Mean tensile stress; PTS: Peak tensile stress; SBP: Systolic blood pressure; SWD: Shear wave dispersion; $S W D_{R}$ : Shear wave dispersion in electrocardiographic $R$ wave; SWE: Shear wave elastography; $S_{W} E_{\mathrm{R}}$ : Shear wave elastic modulus in electrocardiographic R wave; TC: Total cholesterol; TG: Triglycerides; TS: Tensile stress; WT: Wall tension

\section{Acknowledgements}

Not applicable.

Authors' contributions

$\mathrm{ZL}$ and $\mathrm{XL}$ conceived and designed the study. $\mathrm{ZL}, \mathrm{XL}$ and $\mathrm{LD}$ performed the experiments. XL wrote the paper. ZL and LD reviewed and edited the manuscript. All authors read and approved the manuscript. 


\section{Funding}

This work was supported by the Three - year Plan for Clinical Skills and Innovation in Municipal Hospitals (16CR3105B), Shanghai Science and Technology Committee Fund (16411969300), Interdisciplinary Program of Shanghai Jiao Tong University (YG2015MS28), Shanghai Health and Family Planning Commission Fund (201640043) and Shanghai Songjiang District Science and Technology Project (18sjkjgg72 and 18sjkjgg53).

\section{Availability of data and materials}

The datasets are analyzed and are not publicly available, but are available from the corresponding author on reasonable request.

\section{Ethics approval and consent to participate}

Clinical investigations were performed according to the Declaration of Helsinki. The study protocol was approved by the ethics committee of Shanghai General Hospital (2017KY009) and registered with the official website of China Clinical Trial Registration Center (ChiCTR1800016590).

\section{Consent for publication}

Consent for publication was obtained from the patients and family members.

\section{Competing interests}

The authors declare that they have no competing interests.

\section{Author details}

'Department of Echocardiography, Shanghai General Hospital, Shanghai Jiao Tong University School of Medicine, Shanghai 200080, China. ${ }^{2}$ Department of Ultrasound, Shanghai General Hospital, Shanghai Jiao Tong University School of Medicine, 100 Haining Road, Hongkou District, Shanghai 200080, China.

\section{Received: 23 August 2019 Accepted: 8 November 2019}

\section{Published online: 29 November 2019}

\section{References}

1. Maruyama D, Fukuda K, Kataoka H, Morita Y, Nishimura K, Kawamura Y, et al. Evaluation of carotid artery outward remodeling by T1-weighted magnetic resonance imaging in carotid endarterectomy and stenting. J Vasc Surg. 2015:61:1464-71.

2. Kashiwazaki D, Kuwayama N, Akioka N, Noguchi K, Kuroda S. Carotid plaque with expansive arterial remodeling is a risk factor for ischemic complication following carotid artery stenting. Acta Neurochir. 2017;159:1299-304.

3. Ignatenko P, Novikova O, Gostev A, Starodubtsev V, Zeidlits G, Kuznetsov K, et al. Carotid endarterectomy with autoarterial remodeling of bifurcation of the common carotid artery and carotid endarterectomy with patch closure: Comparison of methods. J Stroke Cerebrovasc Dis. 2019;28:741-50.

4. Chironi GN, Simon A, Bokov P, Levenson J. Correction of carotid intimamedia thickness for adaptive dependence on tensile stress: Implication for cardiovascular risk assessment. J Clin Ultrasound. 2009;37:270-5.

5. Berglund JD, Nerem RM, Sambanis A. Viscoelastic testing methodologies for tissue engineered blood vessels. J Biomech Eng. 2005;127:1176-84.

6. Valdez-Jasso D, Bia D, Zócalo Y, Armentano RL, Haider MA, Olufsen MS. Linear and nonlinear viscoelastic modeling of aorta and carotid pressure-area dynamics under in vivo and ex vivo conditions. Ann Biomed Eng. 2011;39:1438-56.

7. Barry CT, Hazard C, Hah Z, Cheng G, Partin A, Mooney RA, et al. Shear wave dispersion in lean versus steatotic rat livers. J Ultrasound Med. 2015;34: 1123-9.

8. Shang J, Wang W, Feng J, Luo GG, Dang Y, Sun J, et al. Carotid plaque stiffness measured with supersonic shear imaging and its correlation with serum homocysteine level in ischemic stroke patients. Korean J Radiol. 2018; 19:15-22

9. Yoon JH, Lee JM, Woo HS, Yu MH, Joo I, Lee ES, et al. Staging of hepatic fibrosis: comparison of magnetic resonance elastography and shear wave elastography in the same individuals. Korean J Radiol. 2013;14:202-12.

10. Li Z, Du L, Wang F, Luo X. Assessment of the arterial stiffness in patients with acute ischemic stroke using longitudinal elasticity modulus measurements obtained with Shear Wave Elastography. Med Ultrason. 2016 18:182-9.

11. Fernandes-Santos C, de Souza ML, Mandarim-de-Lacerda CA. Favorable cardiac and aortic remodeling in olmesartan-treated spontaneously hypertensive rats. Heart Vessel. 2009;24:219-27.
12. Sáez P, García A, Peña E, Gasser TC, Martínez MA. Microstructural quantification of collagen fiber orientations and its integration in constitutive modeling of the porcine carotid artery. Acta Biomater. 2016;33:183-93.

13. Li ZJ, Liu Y, Du LF, Luo XH. Evaluating arterial stiffness in type 2 diabetes patients using ultrasonic radiofrequency. J Huazhong Univ Sci Technolog Med Sci. 2016;36:442-8

14. Gummadi S, Eisenbrey J, Li JZ, Li ZJ, Forsberg F, Lyshchik A, Liu JB. Advances in modern clinical ultrasound. Advanced Ultrasound in Diagnosis and Therapy. 2018;2:51-63.

15. Kokubo Y, Watanabe M, Higashiyama A, Nakao YM, Nakamura F, Miyamoto $Y$. Impact of intima-media thickness progression in the common carotid arteries on the risk of incident cardiovascular disease in the suita study. J Am Heart Assoc. 2018:7:e007720.

16. Luo X, Yang Y, Cao T, Li Z. Differences in left and right carotid intima-media thickness and the associated risk factors. Clin Radiol. 2011:66:393-8.

17. Polak JF, Kronmal RA, Tell GS, O'Leary DH, Savage PJ, Gardin JM, et al. Compensatory increase in common carotid artery diameter. Relation to blood pressure and artery intima-media thickness in older adults. Cardiovascular Health Study. Stroke. 1996;27:2012-5.

18. Carallo C, Tripolino C, De Franceschi MS, Irace C, Xu XY, Gnasso A. Carotid endothelial shear stress reduction with aging is associated with plaque development in twelve years. Atherosclerosis. 2016;251:63-9.

19. Gemignani T, Azevedo RC, Higa CM, Coelho OR, Matos-Souza JR, Nadruz W Jr. Increased popliteal circumferential wall tension induced by orthostatic body posture is associated with local atherosclerotic plaques. Atherosclerosis. 2012;224:118-2.

20. Haga JH, Li YS, Chien S. Molecular basis of the effects of mechanical stretch on vascular smooth muscle cells. J Biomech. 2007:40:947-60.

21. Cunnane EM, Mulvihill JJE, Barrett HE, Hennessy MM, Kavanagh EG, Walsh MT. Mechanical properties and composition of carotid and femoral atherosclerotic plaques: A comparative study. J Biomech. 2016:49:3697-704.

22. Pasquesi SA, Liu Y, Margulies SS. Repeated loading behavior of pediatric porcine common carotid arteries. J Biomech Eng. 2016:138:124502.

23. Soleimani E, Mokhtari-Dizaji M, Fatouraee N, Saberi H. Estimation of biomechanical properties of normal and atherosclerotic common carotid arteries. Cardiovasc Eng Technol. 2019;10:112-23.

24. Yoon JH, Cho IJ, Chang HJ, Sung JM, Lee J, Ryoo H, et al. The value of elastic modulus index as a novel surrogate marker for cardiovascular risk stratification by dimensional speckle-tracking carotid ultrasonography. J Cardiovasc Ultrasound. 2016;24:215-22.

25. Li ZJ, Du LF QY, Liu JB, Luo XH. Ultrasound assessment of intima-media thickness and diameter of carotid arteries in patients undergoing hemodialysis or renal transplantation. Curr Med Sci. 2018;38:727-33.

26. Di Leo N, Venturini L, de Soccio V, Forte V, Lucchetti P, Cerone G, et al. Multiparametric ultrasound evaluation with CEUS and shear wave elastography for carotid plaque risk stratification. J Ultrasound. 2018:21:293-300.

27. Peper ES, Strijkers GJ, Gazzola K, Potters WW, Motaal AG, Luirink IK, et al. Regional assessment of carotid artery pulse wave velocity using compressed sensing accelerated high temporal resolution 2D CINE phase contrast cardiovascular magnetic resonance. J Cardiovasc Magn Reson. 2018;20:86.

28. Zhang Y, Ding H, Wu SD, Fan PL, Li Z, Zeng WJ, Wang WP. Histological Referencefor shear wave Elastography in liver fibrosis: collagen quantification andScoring system. Adv Ultrasound DiagnTher. 2019;3:87-96.

29. Ghigo AR, Wang XF, Armentano R, Fullana JM, Lagree PY. Linear and nonlinear viscoelastic arterial wall models: Application on animals. J Biomech Eng. 2017;139:1-7.

30. Osidak MS, Osidak EO, Akhmanova MA, Domogatsky SP, Domogatskaya AS. Fibrillar, fibril-associated and basement membrane collagens of the arterial wall: architecture, elasticity and remodeling under stress. Curr Pharm Des. 2015:21:1124-33.

31. Khamdaeng T, Luo J, Vappou J, Terdtoon P, Konofagou EE. Arterial stiffness identification of the human carotid artery using the stress-strain relationship in vivo. Ultrasonics. 2012:52:402-11.

32. Wendorff $C$, Wendorff $H$, Kuehnl A, Tsantilas P, Kallmayer M, Eckstein HH, et al. Impact of sex and age on carotid plaque instability in asymptomatic patients-results from the Munich Vascular Biobank. Vasa. 2016:45:411-6.

\section{Publisher's Note}

Springer Nature remains neutral with regard to jurisdictional claims in published maps and institutional affiliations. 